glykano-Hydrolasen am physiologischen Mucopolysaccharid-Stoffwechsel des Tumors möglich, wobei die simultane oder successive Aktion von mindestens drei (Hyaluronatglykanohydrolase, $\beta$-Glucuronidase, $N$-Acetyl- $\beta$-glucosaminidase) wahrscheinlich jedoch mehr Glykosidasen notwendig ist. Hierfür spricht auch die Beobachtung, daß für die $\beta$-Glucuronidase die höchste Aktivität im mucopolysaccharidreichen Randgebiet des Tumors gefunden wurde (19) und daß neben $\operatorname{der} \beta$ Glucuronidase auch andere Glykosidasen im Tumorgewebe nachgewiesen wurden, wie ein die Viskosität von Hyaluronat reduzierendes Prinzip aus Rous-Sarkom (2) und ein Hyaluronat-hydrolysierendes Enzym sowie eine $\beta$-Glucosaminidase ${ }^{1}$ ) aus Asciteszellen (20). Zum anderen lassen die hohen im Walker-Carcino-Sarkom ge- fundenen Enzymaktivitäten daran denken, daß die enzymatische Hydrolyse der Mucopolysaccharide des benachbarten Bindegewebes des Wirtsorganismus das infiltrative expansive Wachstum des Tumors erleichtert, der Tumor also die ebenfalls mucopolysaccharidreiche bindegewebige Abwehrzone des Wirtsorganismus enzymatisch durchbricht.

Die Arbeit wurde durch die Deutsche Forschungsgemeinschaft und den Fonds der Chemischen Industrie in dankenswerter Weise unterstützt. Für die Durchführung eines Teils der enzymatischen Aktivitätsbestimmungen danken wir den Herren O. HoEfELE, G. ReICH und Dr. U. STEIN.

1) In Asciteszellhomogenaten fanden wir eine N-Acetyl- $\boldsymbol{\beta}$-glucosaminidase, die nach Reinigung durch Ammoniumsulfatfällung und Chromatographie in einer spezifischen Aktivität von $0,093 \mathrm{U} / \mathrm{mg}$ Protein erhalten werden konnte.

\title{
Literatur
}

1. Sylvén, B. Aminos ugarcontaining compounds in tumors, in: Balazs, E. und R. W. Jeanloz, The Amino sugars, Vol. II A, Academic Press, New York (1965). - 2. Caputo, A. und M. L. Marcante, Cancer Res. 19, 1010 (1959). ORR, S. F. D., Biochem. biophysica Acta (Amsterdam) 14, 173 (1954). - 3. WARREN, G. H., E. Horenstenn und J. Gray, Arch. Biochem. Biophysics 44, 107 (1953); Merker, H. J., H. Greiling und Th. Günther, Zschr. Krebsforsch. 63, 491 (1960). - 4. Rilex, J. E., The Mast cells, Livingstone, Edinburgh-London (1959). - 5. BUDDECKE, E., W. KröZ und E. Lanka, Hoppe-Seyler's Z. physiol. Chem. 331, 196 (1963); BUDdeCKE, E., Hoppe-Seyler's Z. physiol. Chem. 318, 33 (1960). - 6. Reissig, J. L., J. L. Strominger und L. F. Leloir, J. biol. Chemistry 193, 265 (1951). 7. Buddecke, E. und D. Platr, Hoppe-Seyler's Z. physiol. Chem. 343, 61 (1965). - 8. BUDDECKE, E. und E. Werries, Hoppe-Seyler's Z. physiol. Chem. 340, 257 (1965). - 9. Talalay, P., W. H. Fishman und C. Huggins, J. biol.
Chemistry, 166, 757 (1946). - 10. Anson, M. L., J. gen. Physiol. 22, 79 (1938). - 11. Frimmer, M. und E. Buddecke, Z. Naturforsch. 196, 789 (1964). - 12. GotrschaLK, A. und S. FAZEKAS DE ST. Groth, Biochim. biophysica Acta (Amsterdam) 43, 513 (1960). -13. Barker, S. A., M. StaceY und D. J. Tipper, Nature (London) 184, 68 (1959). - 14. Langley, O. K. und E. J. Ambrose, Nature (London) 204, 53 (1964). - 15. Matheson, A. T. und B. L. Tatrrie, Canad, J. Biochem. 42, 95 (1964). - 16. Jackson, D. S., D. B. Flickinger und J. E. Dunphy, Ann. N. Y. Acad. Sci. 86, 943 (1960). - 17. Lippman, M., Transact. N.Y. Acad. Sci. (Ser. II) 27, 342 (1965). - 18. Fishman, H. W. und A. J. Anlyan, Cancer. Res. 7, 808 (1947). - Fisher, E. R. und B. Fisher, Cancer. Res. 21, 527 (1961). - 19. Fishman, W. H., G. W. Mitchell, P. R. F. Borges, K. T. Ladue und M. Hayashi, Cancer. Res. 16, 118 (1963). - 20. GüNTHER, Th., M. Wenzel und T. Eberhard, diese Z. 3, 76 (1965).
Professor Dr. E. Buddecke, Physiol.-Chem. Institut der Universität 74 Tübingen, Auf dem Schnarrenberg

\section{Beitrag zur Aufklärung des thermischen Verhaltens von Bence-Jones-Proteinen}

\author{
Von H. Hauser, A. Holasek und I. Pascher \\ Aius dem Institut für Physiologische Chemie der Universität Graz (Vorstand: Prof. Dr. A. Holasek)
}

(Eingegangen am 7. März 1966)

Reaktionskinetisch unterscheidet sich die Hitzefällung und Hitzedenaturierung des im Rahmen dieser Arbeit untersuchten Bence-Jones-Proteins („BJP-R“) nicht von der ànderer Eiweißkörper. Eine Dissoziation des BJP-R konnte bei einer Ionenstärke $\mu=0,1$ weder nach dem Erhitzen auf $90-100^{\circ}$ noch bei $\mathrm{pH}$-Werten zwischen 2 und 10 nachgewiesen werden. Bei geringerer Ionenstärke $(\mu=0,01)$ findet man bei $\mathrm{pH}$-Werten unter 4 und über 9 eine Abnahme der Sedimentationskonstanten und eine Verzögerung bei der Gelfiltration. Beide Erscheinungen könnten jedoch auch durch den Ladungseffekt ohne Dissoziation erklärt werden. Da auch beim Ovalbumin die Hitzelöslichkeit mit steigender Temperatur über $100^{\circ}$ zunimmt, muß eine Dissoziation der Bence-Jones-Proteine nicht die Voraussetzung für die Hitzelöslichkeit sein.

Bence-Jones protein (,BJP-R") does not differ from other proteins in its kinetics of heat precipitation and heat denaturation. At an ionic strength $\mu=0.1$, dissociation of BJP-R could not be detected either after heating to $90-100^{\circ}$, or at $\mathrm{pH}$ values between 2 and 10 . At lower ionic strength $(\mu=0.01)$, below $\mathrm{pH} 4$ and above $\mathrm{pH} 9$, there is a decrease in the sedimentation constant and a retardation in gel filtration. Both effects, however, could be explained by changes in electrical charge, without dissociation. Since the temperature solubility of ovalbumin also increases with temperature above $100^{\circ}$, dissociation of Bence-Jones proteins need not be a requirement for heat solubility. 
Im Hinblick auf die enge Beziehung der Bence-JonesProteine (BJP) zu den Immunoglobulinen ist die Frage von besonderem Interesse, ob das eigenartige thermische Verhalten dieser Paraproteine tatsächlich nur auf ihre leichte Spaltbarkeit oder doch auf gewisse Eigenarten ihrer Tertiärstruktur zurückzuführen ist. Die verschiedenen Antikörper besitzen bei sicher nur geringen, wenn überhaupt vorhandenen Unterschieden in der quantitativen Zusammensetzung besondere Oberflächenstrukturen, die es ihnen ermöglichen, sich mit den Antigenen reversibel zu verbinden. Der Beginn der Hitzepräzipitation von $\mathrm{BJP}$ bei Temperaturen ab $45^{\circ}$, die Auflösung des Niederschlages bei Temperaturen über $90^{\circ}$, die leichte „Renaturierbarkeit“ des hier untersuchten BJP-R könnten in irgendeinem Zusammenhang mit der Konfiguration der Immunoglobuline stehen. Unterstützt wird diese Annahme durch die Tatsache, daß die aus 7 s-Globulinen gewonnenen Bruchstücke das gleiche thermische Verhalten zeigen wie die BJP (1). Die bisher von F. W. Putnam (2) und dann auch von anderen Autoren $(3,4)$ untersuchten BJP zeigten alle in der Hitze eine Dissoziation in kleinere Bruchstücke, was zur Erklärung der Hitzelöslichkeit herangezogen wurde. Weitere Versuche von Putnam (5) ergaben, daß viele $\mathrm{BJP}$ bei $\mathrm{pH}$-Werten unter 4 eine Erniedrigung der Sedimentationskonstante zeigen, woraus ebenfalls auf einen Zerfall in Bruchstücke geschlossen werden kann. Es sollte nun untersucht werden, ob sich die Hitzepräzipitationen des BJP-R reaktionskinetisch von der Hitzefällung anderer Proteine wesentlich unterscheidet, ob dieses Paraprotein die gleiche Dissoziierbarkeit zeigt wie die bisher untersuchten BJP und unter welchen Bedingungen sich das einmal denaturierte und gefällte Protein wieder bleibend in Lösung bringen läßt, also anscheinend „renaturiert". Die Eigenschaften dieses als „renaturiert" bezeichneten BJP sollten mit denen des nativen verglichen werden.

\section{Methodik}

Kristallisiertes Bence-Jones.Protein: Nach seiner Herkunft wurde es mit dem Buchstaben R bezeichnet („BJP-R“) (6). Die Einheitlichkeit wurde durch Elektrophorese bei verschiedenen $\mathrm{pH}$-Werten, durch Analyse in der Ultrazentrifuge, durch Chromatographie auf DEAE- und CM-Cellulose und durch Gelfiltration auf Sephadex G 75 bewiesen.

Kristallisiertes Ovalbumin: wurde nach SøRENSEN und Høyrup (7) dargestellt.

\section{Bestimmung der Proteinkonzentration}

$1 \mathrm{mg} \mathrm{BJP}-\mathrm{R} / \mathrm{ml}$ hat bei einer Schichtdicke von $1 \mathrm{~cm}$ und bei $280 \mathrm{~m} \mu$ eine Extinktion von 1,424 $\pm 0,004$. Die Eichung basiert auf der Bestimmung des Trockengewichtes des Eiweißkörpers bei $105^{\circ}$.

\section{Standardbedingungen fïr die Hitzefällung}

$0,2 \mathrm{~g}$ Protein werden in einer $0,02 \mathrm{M}$ Pufferiösung (Acetat, Phosphat, Citrat oder Michaelis) gelöst, die mit Natriumchlorid auf eine Ionenstärke von $\mu=0,1$ gebracht wird. Die Konzentration der Proteinlösung beträgt $0,2 \mathrm{~g} / 100 \mathrm{~m} /$ Lösung. Wenn nicht anders angegeben, werden jeweils $5 \mathrm{ml}$ der Eiweißlösung $15 \mathrm{Min}$. auf der gewünschten Temperatur gehalten, rasch abgekühlt und bei 5000 U./Min. zentrifugiert. Im Überstehenden wird die Eiweißkonzentration photometrisch bestimmt.

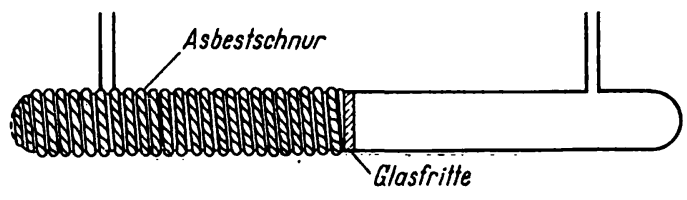

Abb. 1

Gerät zur Filtration bei Temperaturen über $100^{\circ}$

Die Bestimmung der Hitzelöslichkeit bei Temperaturen über $100^{\circ}$ erfolgt in einem $10 \mathrm{~cm}$ langen, $1 \mathrm{~cm}$ breiten Glastohr, das in der Mitte durch eine Glasfritte $G 2$ geteilt ist (Abb. 1). Die eine Hälfte ist mit einer Asbestschnur umwickelt. In diese Hälfte wird die Eiweißlösung so eingefüllt, daß sie die Fritte nicht benetzt. Dann werden die Öffnungen des Rohres verschmolzen und das ganze Rohr 15 Min. waagrecht in einem Olbad auf der Versuchstemperatur gehalten. Anschließend wird das Rohr aus dem Ölbad genommen und sofort in die vertikale Lage gekippt. Auf diese Weise wird die Lösung durch die Glasfritte gesaugt und vom Niederschlag abfiltriert. Durch Zusatz von Natronlauge wird der beim $\mathrm{Ab}$ kühlen entstehende Niederschlag gelöst und die Proteinkonzentration photometrisch bestimmt.

Die Ladungszahl des BJP-R wird so bestimmt, daß die ausgiebig gegen aqua dest. dialysierte Eiweißlösung schrittweise mit 0,1 N Salzsäure versetzt wैird. Aus der zugesetzten Menge an Säure und dem jeweiligen $\mathrm{pH}$-Wert läßt sich die Ladungszahl, d. h. die Anzahl von Ladungen pro Molekül, berechnen.

Die Sedimentationskonstante wird in einer luftgetriebenen Ultrazentrifuge Type U $50 \mathrm{~L}$ der Fa. Phywe, Göttingen, bei $50000 \mathrm{U}$./ Min. bestimmt. Die Beobachtung des Sedimentationsvorganges erfolgt mit einer Schrägoptik nach Pbilpot-Svensson.

Die Diffusionskonstante wird in der Unterschichtungszelle der Diffusionseinrichtung zur Phywe-Ultrazentrifuge bestimmt.

Das partielle spezifische Volumen wird mit Hilfe von Präzisionspyknometern von 10 bzw. $3 \mathrm{~m} l$ Fassungsvermögen ermittelt (8).

\section{Ergebnisse}

\section{Reaktionskinetik der Hitzepräzipitation}

Für die folgenden Untersuchungen war es zunächst zweckmäßig zu ermitteln, inwiefern eine nicht zur Fällung führende Denaturierung reversibel ist. Eine auf pH 9,1 gebrachte BJP-R-Lösung zeigte beim Erhitzen kẹine Fällung. Durch Einpipettieren dieser erhitzten Lösung in einen Fällungspuffer $(0,1 \mathrm{~m}$ Acetatpuffer $\mathrm{pH} 4,6 ; 1 \mathrm{M}$ an Natriumchlorid) kam es zur Präzipitation. Wie Abbildung 2 zeigt, lag die optimale Versuchstemperatur zur Ermittlung der Reversibilität zwischen $80^{\circ}$ und $90^{\circ}$. Die Proteinlösung in einem Borat-PhosphatPuffer von pH 9,1 wurde 15 Min. auf $80^{\circ}$ erhitzt und anschließend rasch auf Zimmertemperatur abgekühlt. In

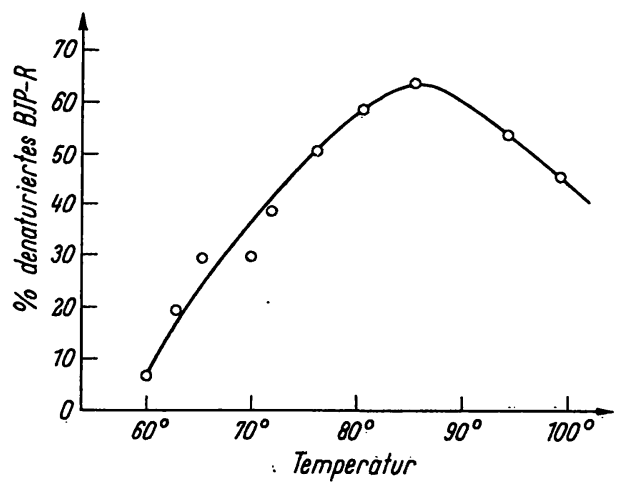

Abb. 2

Prozent denaturiertes bzw. fällbares $B J P-R$ in Abhängigkeit von der Temperatur ( $\mathrm{pH}=9,1)$ 


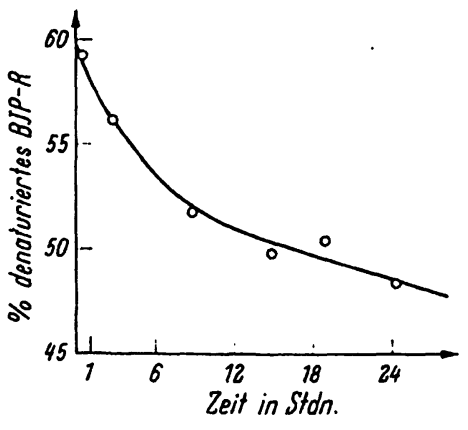

Abb. 3

Fällbarkeit des bei pH 9,1 hitzedenaturierten BJP-R in Abhängigkeit von der Aufbewahrungszeit bei Zimmertemperatur

genauen Zeitabständen wurde jeweils $1 \mathrm{ml}$ dieser Lösung in $5 \mathrm{ml}$ Fällungspuffer pipettiert und der Niederschlag abzentrifugiert.

Die Abbildung 3 zeigt die gefällte Proteinmenge, aufgetragen gegen die Aufbewahrungszeit bei $20^{\circ}$. Ein Teil der denaturierten BJP-R-Lösung wurde 8 Wochen bei Zimmertemperatur stehengelassen; es ließen sich nach dieser Zeit unter den gegebenen Bedingungen nur etwa $4 \%$ fällen. Die Versuche zeigten, daß die Denaturierung an und für sich reversibel ist, $\mathrm{da} ß$ aber innerhalb der Versuchszeit von etwa 1 Stunde keine nennenswerte „Renaturierung“ eintritt. Dies konnte auch durch einen zweiten Versuch gezeigt werden. Aus einer unter Standardbedingungen bei $\mathrm{pH} 4,6$ auf $60^{\circ}$ erhitzten BJP-R-Lösung fielen $93 \%$ des Proteins aus. Kühlte man diese Lösung mit dem Niederschlag auf $53^{\circ}$ ab und ließ sie bei dieser Temperatur mehrere Stunden stehen, so nahm die Niederschlagsmenge nur auf $88 \%$ ab. Bei $53^{\circ}$ fand man jedoch unter Standardbedingungen eine 67-proz. Fällung. Innerhalb einer Versuchsdauer von 7 Stdn. kam es also zu keiner Einstellung des Gleichgewichtes; die Niederschlagsmenge nahm nur wenig ab.

Daß die durch Hitze eintretende Proteindenaturierung und Enzyminaktivierung Reaktionen erster Ordnung sind, wurde an vielen Beispielen gezeigt $(9,10)$, obwohl oft beträchtliche Abweichungen von den für Reaktionen erster Ordnung charakteristischen Konstanten auftreten (11). Auch die Hitzepräzipitation des BJP-R zeigte die Kriterien einer Reaktion erster Ordnung. Die Geschwindigkeit der Hitzefällung, ausgedrückt als prozentuale Änderung der Eiweißkonzentration, ist nämlich unabhängig von der Konzentration der Lösung. In Abbildung 4 wurde die Menge des bei pH 4,6 unter Standardbedingungen bei $53,0^{\circ}$ bzw. 60,1 ${ }^{\circ}$ aus 0,2-proz. und 0,1-proz. Lösung gefällten Proteins in Abhängigkeit von der Zeit aufgetragen.

Zur Ermittlung der Reaktionsordnung $n$ und Geschwindigkeitskonstanten $\mathrm{k}$ wurden Hitzefällungen bei $53,0^{\circ}, 54,0^{\circ}, 56,3^{\circ}$ und $60,1^{\circ}$ bei $\mathrm{pH} 4,6$ unter Standardbedingungen durchgeführt. $\mathrm{Zu}$ diesem $\mathrm{Zweck}$ wurden $50 \mathrm{ml}$ Acetatpuffer $\mathrm{pH} \mathrm{4,6}$ auf die gewünschte Temperatur erwärmt. Nach Erreichung der Temperatur wurden $2 \mathrm{ml}$ konzentrierter vorgewärmter BJP-R-Lösung einpipettiert. Wenn die Pipette halb entleert war, begann

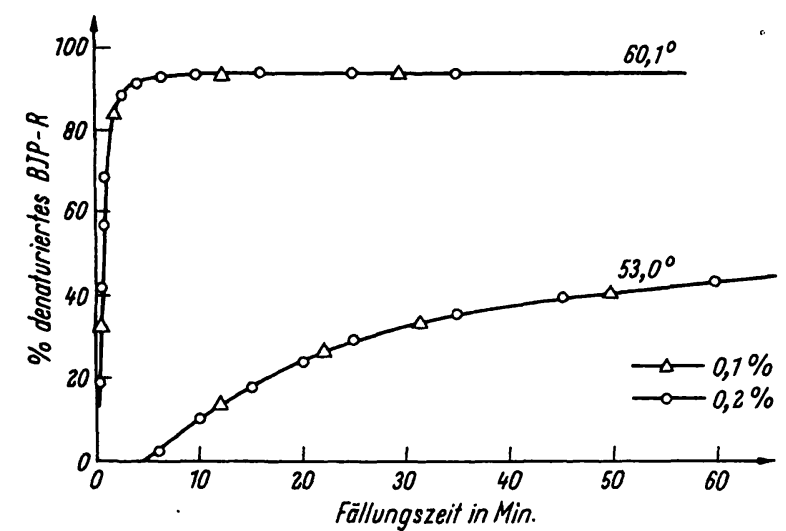

Abb. 4

Prozent gefälltes BJP-R in Abhängigkcit von der Erhitzungsdauer bei zwei Temperaturen und Konzentrationen; $(\mathrm{pH}=4,6)$

die Zeitmessung. Die Eiweißkonzentration und die Pufferlösung wurden so gewählt, daß nach dem Mischen die Standardbedingungen in bezug auf beide Konzentrationen gegeben waren. In angepaßten Zeitabständen wurden $4 \mathrm{~m} /$ des Reaktionsgemisches in eisgekühlte Zentrifugengläser pipettiert. Nach halbstündigem Stehen bei $0^{\circ}$ wurde der Niederschlag abzentrifugiert und in der überstehenden Lösung die Eiweißkonzentration photometrisch bestimmt.

In Abbildung 5 ist die Menge des präzipitierten BJP als Bruchteil der Ausgangskonzentration in Abhängigkeit von der Erhitzungsdauer für die vier Temperaturen wiedergegeben.

Behandelt man die Versuchsdaten der Abbildung 5 in der Art, daß man den allgemeinen Zusammenhang zwischen Geschwindigkeit $v$ und Konzentration $\mathrm{c}$ - nämlich $\mathrm{v}=\mathrm{kc}^{\mathrm{n}}$ - zur Bestimmung der Reaktionsordnung $n$ verwendet, so müssen die Kurven in Abbildung 5 differenziert werden. Trägt man in einem Diagramm log $\nabla$ gegen $\log \mathrm{c}$ auf, so erhält man für alle 4 Temperaturen eine Gerade. Die Steigung der Geraden ergibt die Reaktionsordnung $n$ :

$$
\begin{array}{ll}
53,0^{\circ} \ldots \ldots n=1,53 & 56,3^{\circ} \ldots \ldots n=1,01 \\
54,0^{\circ} \ldots \ldots n=1,06 & 60,1^{\circ} \ldots \ldots n=1,25
\end{array}
$$

Die Geschwindigkeit der Hitzefällung ist proportional der Differenz zwischen Eiweißkonzentration zur Versuchszeit und der in Lösung befindlichen Eiweißmenge nach Einstellung des Gleichgewichtes, d. h. bei maximaler Fällung (bei gegebener Temperatur). $\mathbf{x}=$ Menge des gefällten Proteins in $\%$ zur Zeit $t$;

$\mathbf{x}_{\max }=$ maximale Menge des gefällten Proteins in \% nach Erreichung des Gleichgewichtes;

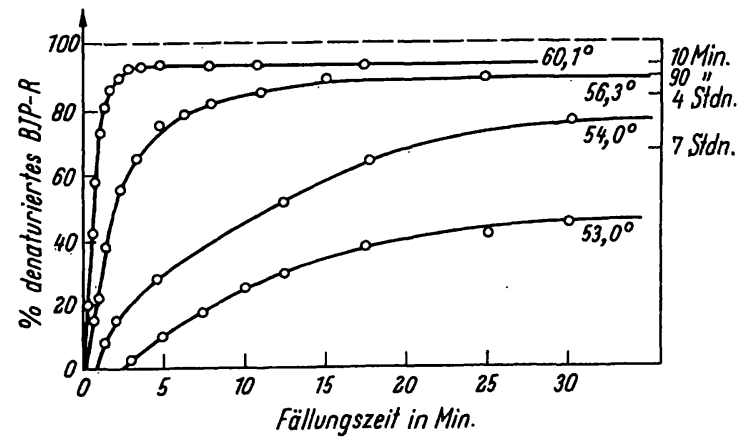

Abb. 5

Prozent gefälltes BJP-R in Abhängigkeit von der Erhitzungsdauer bei vier verschiedenen Temperaturen $(\mathrm{pH}=4,6)$ 
$a=$ Konzentration des löslichen Proteins zur Zeit $\mathrm{t}$ in \% der ursprünglichen Konzentration; $a=100-x$;

$a_{e}=$ Gleichgewichtskonzentration des in Lösung befindlichen Proteins in $\%$ der ursprünglichen Konzentration; $a_{e}=$ $100-x_{\max }$

Trägt man nun $\log \left(x_{\max }-x\right)$ gegen die Zeit auf, so liegen die Punkte für eine gegebene Temperatur weitgehend auf einer $\mathrm{Ge}$ raden (Abb. 6). Die Geschwindigkeitskonstante für eine bestimmte Temperatur erhält man aus der Steigung der Geraden:

$$
\mathrm{k}=\frac{-2,3 \log \left(\mathrm{x}_{\max }-\mathrm{x}\right)}{\Delta \mathrm{t}}=\frac{-2,3 \log \left(\mathrm{a}-\mathrm{a}_{\mathrm{e}}\right)}{\Delta \mathrm{t}}
$$

Trägt man die für die vier verschiedenen Temperaturen gefundenen ln $k$ Werte gegen $1 / T$ auf, so erhält man eine Gerade (Abb. 7).

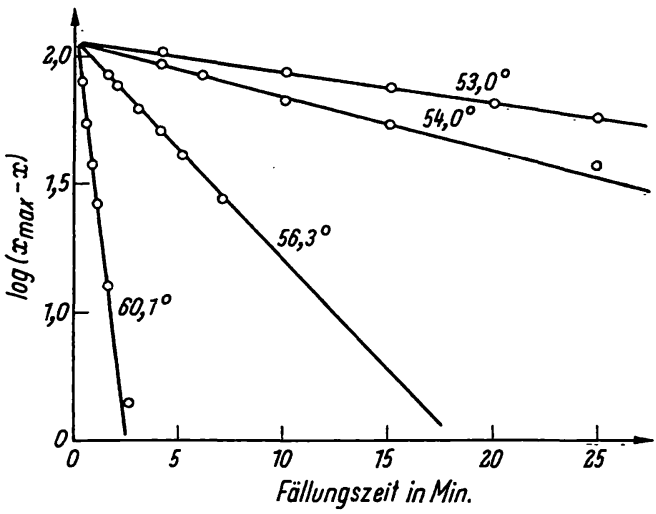

Abb. 6

$\log \left(x_{\max }-\mathrm{x}\right)$ aufgetragen gegen die Dauer des Erhitzens

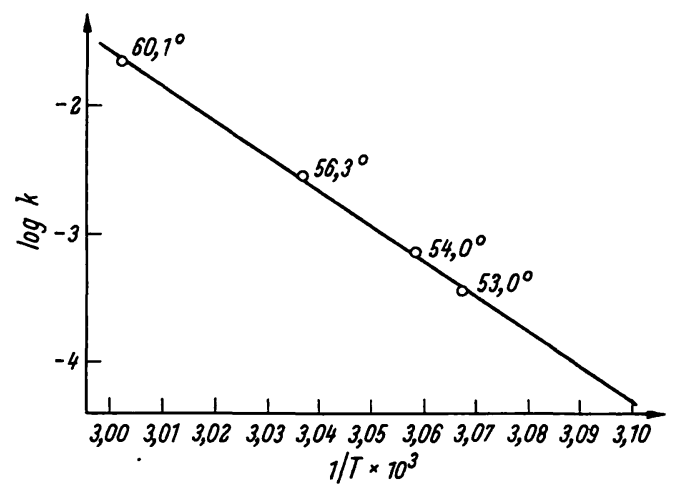

Abb. 7

Logarithmus der Geschwindigkeitskonstanten gegen den reziproken Wert der absoluten Temperatur
Die Steigung dieser Geraden ist nach der Gleichung von Arrbenius $\ln \mathrm{k}=\mathrm{A}-\frac{\mathrm{E}}{\mathrm{RT}}$ gleich $-\mathrm{E} / \mathrm{R}$. Daraus kann dieAktivierungsenergie für die Hitzefällung berechnet werden:

$$
\mathrm{E}=\frac{-2,3 \mathrm{R} \Delta \log \mathrm{k}}{\Delta 1 / \mathrm{T}}=124000 \mathrm{cal}
$$

Nach ExRING (12) ist nicht die Aktivierungsenergie E sondern die Ånderung der freien Energie $\Delta \mathrm{F}$ für die Geschwindigkeit der Denaturierung maßgebend. Nach seiner „theory of absolute reaction rate" ist

$$
\mathrm{k}=\frac{\mathrm{K}}{\mathrm{h}} \mathrm{T} \mathrm{e}^{-\frac{\Delta \mathrm{F}}{\mathrm{RT}}}
$$

$\mathrm{K}=$ Boltzmannsche Konstante $\mathrm{h}=$ Plancksches Wirkungsquantum

Daraus läßt sich die Änderung der freien Energie $\Delta \mathrm{F}$ für die Aktivierungsreaktion (nativ $\rightarrow$ aktivierter Zustand) berechnen:

$$
\Delta \mathrm{F}=\mathrm{RT}\left(\ln \frac{\mathrm{K}}{\mathrm{h}}+\ln \mathrm{T}-\ln \mathrm{k}\right)
$$

Entșprechend der hohen Aktivierungsenergie, die für Proteindenaturierungen charakteristisch ist (13), ist auch eine entsprechend große Entropieänderung zu erwarten. - Durch Einsetzen in die Gibbs-Helmboltzsche Gleichung $\Delta \mathrm{H}=\Delta \mathrm{F}+\Delta \mathrm{ST}$ und aus $\Delta \mathrm{H}=\mathrm{E}-\mathrm{RT}$ läßt sich die Entropiezuñahme $\Delta \mathrm{S}$ für die Denaturierung berechnen. In der untenstehenden Tabelle sind die thermodynamischen Daten zusammengefaßt.

\section{Einfluß des $p H$-Wertes auf die Molekiulgröße}

BJP-R wurde in verschiedenen Pufferlösungen von $\mathrm{pH} 2$ bis $\mathrm{pH} 12$ gelöst und bei einer Ionenstärke von 0,1 in der Ultrazentrifuge untersucht. Wie aus der Abbildung 8 ersichtlich ist, steigt bei pH-Werten unter 4 und über 8 die Sedimentationskonstante von 3,61 s auf 3,75 s, also um $4 \%$ an. Bei pH-Werten unter 2,5 wurden die Gradienten sehr breit; außerdem trat eine zweite schnellere Fraktion, entsprechend der etwa vierfachen Molekülgröße (7,5 bis $8 \mathrm{~s})$ auf. Auf der alkalischen Seite fiel die Sedimentationskonstante bei $\mathrm{pH}$-Werten über $10 \mathrm{ab}$. Die gestrichelte Kurve der Abbildung 8 zeigt die Abhängigkeit der Sedimentationskonstanten vom $\mathrm{pH}$-Wert bei einerIonenstärke unter 0,01. Die Abnahme der Sedimentationskonstanten bei pH-Werten unter 4 bzw. über 9 könnte bei geringen Ionenstärken nur auf einen Ladungseffekt zurückzuführen sein. Dieser führt zu einer Abnahme der Sedimentationsgeschwindigkeit. Ob neben diesem Ladungseffekt auch eine Dissoziation eintritt, konnte auf diese Art nicht geklärt werden.

\begin{tabular}{|c|c|c|c|c|}
\hline$\Delta \mathrm{H}=\mathrm{E}-\mathrm{RT}$ & 123,350 & 123,350 & 123,340 & 123,330 \\
\hline$\Delta \mathrm{F}=4,58 \mathrm{~T}(10,318+\log \mathrm{T}-\log \mathrm{k})$ & 24,300 & 23,900 & 23,00 & 22,100 \\
\hline$\Delta \mathrm{S}=\frac{\Delta \mathrm{M}-\Delta \mathrm{T}}{\mathrm{T}}$ & 304 & 304 & 304 & 304 \\
\hline
\end{tabular}

\begin{tabular}{lcccc}
\hline Fällungs-Temp. in ${ }^{\circ} \mathrm{C}\left({ }^{\circ} \mathrm{K}\right)$ & $53,0(326,0)$ & $54,0(327,0)$ & $56,3(329,3)$ & $60,1(333,1)$ \\
\hline $1 / \mathrm{T} \cdot 10^{3}$ & 3,0675 & 3,0580 & 3,0364 & 3,0020 \\
Max. Fällung in \% & 67 & 83 & 91 & 93 \\
k & 0,000368 & 0,000720 & 0,000291 & 0,022 \\
$\log \mathrm{k}$ & $-3,434$ & $-3,143$ & $-2,546$ & $-1,658$ \\
\hline
\end{tabular}

Aktivierungs-Energie E

124000 


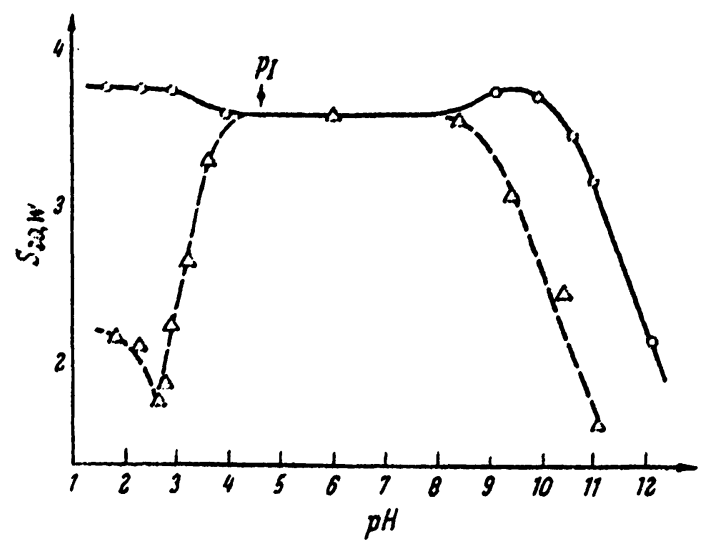

Abb. 8

Anderung der Sedimentationskonstante des BJP-R mit dem plH-Wcrt;

..... : Ioncnstärke $\mu=0,1 ; \ldots .$. : Ioncnstärke $\mu<0,01$

Auch bei der Gelfiltration auf Sephadex $G 75$ mit $0,1 \mathrm{~N}$ Essigsäurc konnte cin gegenüber der I.ösung mit höherer Jonenstärke ver\%ögertes Austreten aus der Säule beobachtet werden (Abb. 9). Die gestrichelte Linic der Abbilclung 9 r.cigt dic Elution von BJP-R mit cincm Acetatpuffer $\mathrm{pH} 4,6, \mu=0,1$ oder mit $0,1 \mathrm{~N}$ Essigsäure hei Zusat\% von Natriumchlorid. Dic Blution mit 0,1 N Essigsäure (ausgezogenc Kurve) ist veřögert und zeigt eincn asymmetrischen Verlauf. Demnach ist auch hicr entweder cin Bremseffekt vorhanden oder cine Spaltung bei pH-Werten unter 4 und über 9 nur bei geringer Ioncnstärke anzunchmen.

Die Versuche bringen keinen schlüssigen Beweis, daß das BJP-R bei Ionenstärken $\mu=0,01$ unterhalb von pH 4 und über pH 9 in Bruchstücke gespalten wird. Dennoch kommt es zu einer Veränderung, die man als Denaturicrung bezcichnen muß: Werden dic klaren Jösungen durch Zusatz von Puffer rasch auf den isoclcktrischen Punkt gebracht, so kommt es zur Koagulation. Bringt man dic Jösungen jedoch langsam, z. B. durch Dialyse, auf den isoelektrischen Punkt, so bleibt der Eiweißkörper in Lösung.

\section{Einflußs des Errbitzens auf die Molekiilgröße}

Wurde cine 1-proz. Lösung von BJP-R in einem $0,005 \mathrm{M}$ Phosphatpuffer pH 6,8 auf $80^{\circ} \mathrm{b} \% \mathrm{w} .100^{\circ}$ crhitzt, so crfolgte nur cine Denaturicrung aber kcinc Fällung. Untersuchtc man die abgekühlte Lösung in der Ultrazentrifuge, so fand man bej der auf $80^{\circ}$ crhitzten Lösung einen symmetrischen Proteingradienten mit cincr Sedimentationskonstanten von $3,75 \mathrm{~s}$. Dic auf $100^{\circ}$ exhitzte Jösung zeigte zwci Gradienten: cinen mit 3,75 s und einen mit $15 \mathrm{~s}$.

Wurde die auf $100^{\circ}$ erhit $/ t e$ und abgekühlte Lösung mit Acetatpuffer rasch auf den $\mathrm{pH}$-Wert 4,6 gebracht, so ficl der Großteil des Proteins aus. Der in Lösung gebliebene 'Tcil des Proteins zeigte in der Ultrazentrifuge eine Sedimentationskonstante von 3,72 s. Dialysierte man das bei $60^{\circ}$ und cinem $\mathrm{pH}-$ Wert von 4,6 gefällte Protein gegen Puffer, deren pH-Werte klciner als 4 oder größer als 6 waren, so löste sich das koagulicrte Eiwciß vollständig

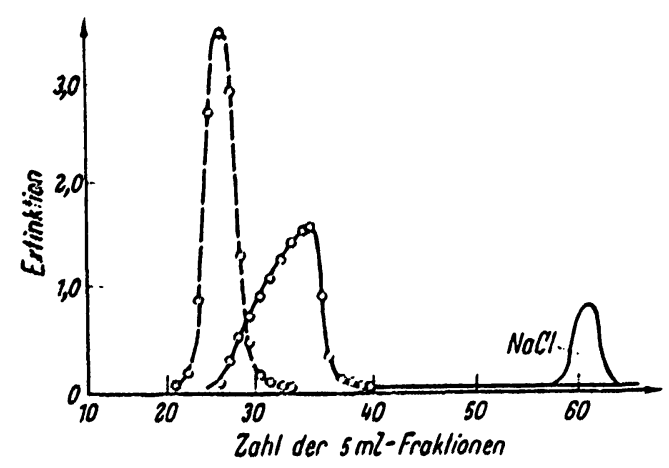

Abb. 9

Gelfiltration des BJP-R auf cincr Scphadex $G 75$ Säulc. ........ : $0,1 \mathrm{~N}$ Bssigsäure;.....$: 0,1 \mathrm{~N}$ Essigsäure $0,1 \mathrm{~N}$ an Natriumchlorid oder Acetatpuffer $\mathrm{pH} \div 4,6$; lonenstärke $\mu=0,1$

auf. Wenn man dic klare Lösung durch Dialyse langsam auf den pH-Wert des isoelektrischen Punktes (pH 4, 6) brachte, so blicb das Protcin fast vollständig in Lösung. In der Ultrazentrifuge \%cigte diese I_ösung cinen cinhcitlichen, symmetrischen Gradienten mit cincr Sedimentationskonstante von $3,79 \mathrm{~s}$.

\section{Eigenschaften des, "renalurierlen" $B J P-\Omega$}

Das hicr untersuchte $B J P-R$, welches nach cince der oben genannten Methoden mit oder ohne Fällung denaturiert und beim isoclektrischen Punkt wieder in J_ösung gebracht wurde, war papicrelcktrophoretisch und bei der Chromatographic cinheitlich. Es «cigte das gleiche thermische Verhalten wic das ursprüngliche Protcin und die gleiche Präzipitationskurve in Abhängigkcit von pH-Wert und Temperatur. Nur die Sedimentationskonstante in 1-proz. I.ösung $s_{20}=3,75 \mathrm{~s}$ und dic Diffusionskonstante $D_{20}=7,42 \cdot 10^{-7} \mathrm{~cm}^{2} / \mathrm{Sck}$. waren um rund $3,5 \%$ von den Werten des nativen $B J P-R$ verschieden. $\mathrm{Da}$ durch dic Denaturierung das particlle spexifische Volumon nicht verändert wurde $\left(V_{20}=\right.$ 0,735), muß cine Zunahme des Molekulargcwichtes von 43100 auf 46500 angenommen werden. Diese Erhöhung ist wahrscheinlich auf cinen geringen Anteil von Molckülaggregaten «urück»uführen.

\section{Bestimmung der Ladungszabl des BJP-R}

Dic in $\Lambda$ bbildung 10 gebrachte Kurve \%eigt die durch Titration mit $0,1 \mathrm{~N}$ Salzsäurc crmitteltc Ladungszahl/ Molckül in Abhängigkeit vom pH-Wcrt. Dic Jaadungszahl wurde auf den isoclektrischen Punkt (pH 4,6) als Nullpunkt bezogen. Bci den pH-Werten 4 und 6 , bei denen das B JP-R nicht mehr gefällt warde, lag die gleiche Anzahl von Ladungen, nämlich $+7 \mathrm{bzw} .-7$ vor. $\mathrm{Bci}$ etwa 6 gleichnamigen I-adungen wurde bei $60^{\circ}$ nur die halbe Proteinmenge gefällt. Die abstoßenden Kräfte diescr 6 Ladungen stehen also im Gleichgewicht mit den Bindungskräften, wclche das Eiweißmolckül im Niederschlag \%usammenhalten.

Behandelt man das BJP-Molekül als Kugel, über deren Oberilüche dic Ladungen gleichmäßig verteilt sind, so kann dic freic elcktrische Encrgic $F$ der geladenen Kugel in einem Lösungsmittel der Di- 


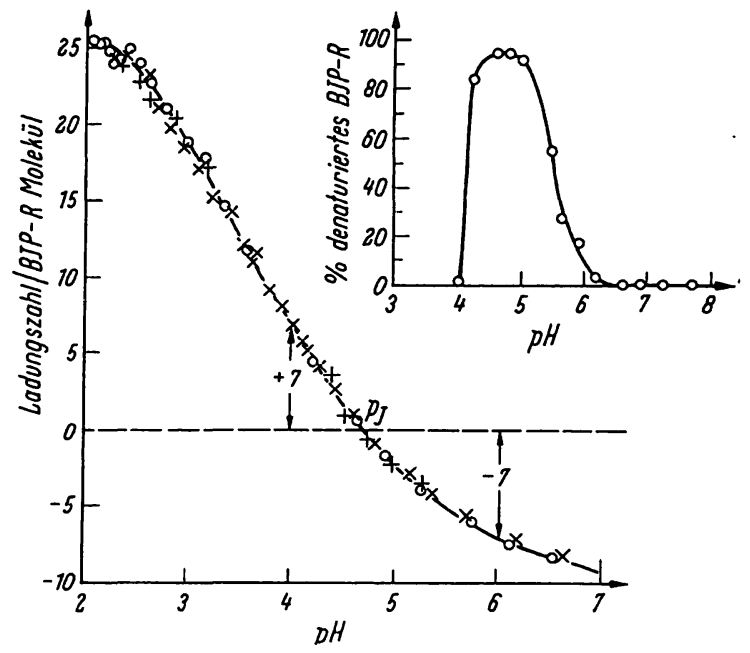

Abb. 10

Ladungszahl des BJP- $\mathrm{R}$ in Abhängigkeit vom $\mathrm{pH}-$ Wert; kleine Kurve: Prozent gefälltes BJP-R in Abhängigkeit vom $\mathrm{pH}$-Wert (Standardbedingungen)

elektrizitätskonstante $\mathrm{D}$ und der Ionenstärke $\mu$ nach SCATCHARD (14) berechnet werden:

$$
\mathrm{F}=\frac{\mathrm{Nz} z^{2} \mathrm{e}^{2}}{2 \mathrm{D}}\left[\frac{1}{\mathrm{~b}}-\frac{\mathrm{K}}{1+\mathrm{Ka}}\right]
$$

$\mathrm{N}=6,02 \cdot 10^{23} ; \mathrm{e}=4,8 \cdot 10^{-10}$ e. stat. E.; $\mathrm{K}=0,329 \cdot 10^{8} \mu$; $\mu=$ Ionenstärke $=0,1 ; \mathrm{D}=$ Dielektrizitätskonstante; für $\mathrm{H}_{2} \mathrm{O}: \mathrm{D}$ $=81 ; z=$ Ladungszahl $=6 ; b=$ Radius des Proteins: für BJP-R $23,3 \cdot 10^{-8}$.

Diese Formel ergibt für die freie elektrische Energie des BJP-R-Moleküls den Wert $\mathrm{F}=4,55 \cdot 10^{10} \mathrm{erg}=1100$ cal. Diese Energie entspricht der mittleren kinetischen Energie E der Moleküle bei $100^{\circ}$ (1120 cal.).

\section{Löslichleeit des Ovalbumins bei Temperaturen über $100^{\circ}$}

Die Abbildung 11 zeigt die Löslichkeit des Ovalbumins, welche mit der eingangs beschriebenen Methode in einem geschlossenen, mit einer Fritte versehenen Röhrchen ermittelt wurde. Die Fällung wurde unter Standardbedingungen bei $\mathrm{pH} \mathrm{4,6} \mathrm{durchgeführt.} \mathrm{Zu} \mathrm{Vergleichs-}$ zwecken wurden die von Bernier und Putnam (15) beschriebenen Versuche über die Löslichkeit von Proteinen bei Temperaturen über $100^{\circ}$ in einem Schmelzpunktsröhrchen wiederholt. Die Temperatur wurde dabei um $1^{\circ}$ pro Minute gesteigert. Unter diesen Bedingungen löst sich das BJP-R bei $107^{\circ}$ und das Ovalbumin bei $170^{\circ}$ vollständig auf.

\section{Diskussion}

Die Ergebnisse sprechen dafür, daß sich das BJP-R bezüglich der Hitzefällung und der Hitzelöslichkeit nicht prinzipiell, sondern nur graduell von anderen Proteinen unterscheidet. Reaktionskinetische Untersuchungen der Denaturierung und Fällung in der Hitze zeigen eine Übereinstimmung mit den bisher untersuchten tierischen Proteinen. Nur das Eintreten der Fällụng bei relativ niedrigen Temperaturen - etwa über $50^{\circ}$ - und die sich zwar langsam einstellende, aber deutliche Reversibilität der Hitzepräzipitation sind, verglichen mit anderen Eiweißkörpern des Serums, als Besonderheit hervor-

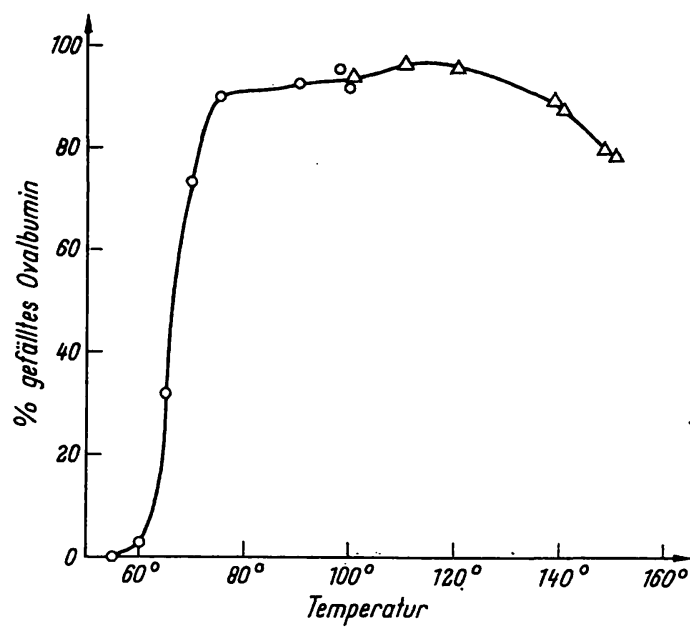

Abb. 11

Prozent gefälltes Ovalbumin in Abhängigkeit von der Temperatur ( $\mathrm{pH}=4,6$; Standardbedingungen)

zuheben. Die exakte Übereinstimmung zwischen der berechneten freien elektrischen Energie des BJP-R-Moleküls und der mittleren kinetischen Energie des Moleküls bei $100^{\circ} \mathrm{muß}$ in Anbetracht der der Berechnung zugrunde liegenden Vereinfachung als Zufall gewertet werden. Die Berechnung sollte nur einen Anhaltspunkt über die Bindungskräfte zwischen den Molekülen des Koagulates geben und die Frage klären, ob die thermische Energie der Moleküle bei Temperaturen um $100^{\circ}$ in dieselbe Größenordnung fällt.

Beim BJP-R konnte weder nach dem Erhitzen noch bei $\mathrm{pH}-$ Werten $\mathrm{zwischen} 2$ und 10 außerhalb des isoelektrischen Punktes und einer Ionenstärke von 0,1 eine Dissoziation nachgewiesen werden. Der bei $\mathrm{pH}-$ Werten $>10$ auftretende Zerfall könnte bereits die Folge einer hydrolytischen Spaltung sein, die bei so hohen $\mathrm{pH}$ Werten nicht ausgeschlossen werden kann. Bei einer Ionenstärke von 0,01 nimmt die Sedimentationskonstante bei $\mathrm{pH}<4$ und $>9$ stark ab, was aber auch ausschließlich die Folge eines Ladungseffektes sein kann. Dieser Ladungseffekt, der bei $\mathrm{pH}$-Werten außerhalb des isoelektrischen Punktes und geringer Ionenstärke auftritt, führt bei unveränderter Molekülgröße zu einer Verkleinerung der Sedimentationskonstante. Der 'Versuch, eine Dissoziation bei geringer Ionenstärke durch Gelfiltration auf Sephadex G 75 sicher nachzuweisen, ist nicht gelungen. Eluiert man mit einem Lösungsmittel $\mathrm{pH} 3, \mu=0,1$, so tritt die Proteinfraktion an derselben Stelle aus wie bei der Elution mit Acetatpuffer $\mathrm{pH} 4,6$, $\mu=0,1$. Bei geringer Ionenstärke $(\mu=0,01)$ und $\mathrm{pH} 3$ beobachtet man einen verzögerten Austritt der Proteinfraktion. Die Elutionskurve zeigt einen asymmetrischen Verlauf. Das verzögerte Austreten der Proteinfraktion kann auch in diesem Falle durch einen Ladungseffekt bedingt sein. Eine Dissoziation bei geringer Ionenstärke und $\mathrm{pH}$-Werten $<4$ und $>9$ konnte demnach nicht mit Sicherheit ausgeschlossen oder nachgewiesen werden. Beim BJP-R kann eine leichte Dissoziation nicht als Erklärung für die Hitzelöslichkeit bei Temperaturen über 
$90^{\circ}$ und einer Ionenstärke von 0,1 herangezogen werden. Die Auflösung des Hitzekoagulates beruht im Falle dieses BJP auf einer Kompensation der intermolekularen Bindungskräfte im Koagulat durch die thermische Energie der Moleküle. Diese Erklärung der Hitzelöslichkeit ließ vermuten; daß die Auflösung von Hitzefällungen bei Temperaturen über $90^{\circ}$ nicht auf BJP beschränkt ist, sondern allgemein bei Proteinen beobachtet werden kann und lediglich in Abhängigkeit von Molekülgröße und -struktur bei mehr oder minder hohen Temperaturen cintritt. Die Versuche mit Ovalbumin und die Untersuchungen von Putnam bestätigen dies. $\mathrm{Da}$ Ovalbumin und viele andere Proteine zwar in der Hitze löslich sind, jedoch meist nicht dissoziieren, ist die Dissoziation der BJP zur Erklärung der Hitzelöslichkeit nicht eine notwendige Voraussetzung.

Die leichte „Renaturierbarkeit" des BJP-R ist einer näheren Untersuchung wert. Es hat sich gezeigt, daß das durch Hitze oder durch extreme $\mathrm{pH}$-Werte denaturierte BJP-R anscheinend seine ursprüngliche Molekülform annimmt, wenn es langsam in die Nähe des isoelektrischen Punktes gebracht wird oder bei diesem pH-Wert länger stehengelassen wird.

\title{
Literatur
}

1. Edelman, G. M. und J. A. Gally, J. Exper. Med. 116, 207 (1962). - 2. Putnam, F. W. und P. Stelbs, J. biol. Chemistry 203, 347 (1953). - 3. Caputo, A., Oncologia 12, 203 (1959). - 4. HeIMER, R., E. R. SCHWARTZ, R. L. ENGLE und K. R. Woods, Biochemistry 2, 1380 (1963). - 5. Putnast, F. W., C. W. Easley, L. T. Lynn, A. E. Ritchil und R. A. Phelps, Arch. Biochem. Biophysics 83, 115 (1959). - 6. Holasek, A., I. PASCher und H. Hauser, Mh. Chem. 92, 463 (1961). - 7. Sørensen, S. P. L. und M. Høyrup, Hoppe-Seyler's Z. physiol. Chem. 103, 15 (1918). - 8. Diermayr, E. und A. Holasek, Microchem. J. (New York)
10, 231 (1966). - 9. Kunitz, M., J. Gen. Physiol. 32, 241 (1949). 10. Eisenberg, M. A. und G. W. Schwert, J. Gen. Physiol. 34, 583 (1950). - 11. Casey, E. J. und K. I. LaIdLER, J. Amer. chem. Soc. 73, 1455 (1951). - 12. EYring, H. I., J. chem. Physics 3, 107 (1935). - 13. Stearn, A. E., Advances in Enzymol. 9, 25 (1945). 14. Scatchard, S. in Cohn Edsall, Proteins Amino Acids and Peptids, S. 445, 473-75, Reinhold Publ. Corp., New York (1943). - 15. Bernier, G. M. und F. W. Putnan, Biochim. biophysica Acta (Amsterdam) 86, 295 (1964).

Professor Dr. A. Holasek

Physiolog.-chem. Institut der Universität Graz (Österreich) Universitätsplatz 2

\section{Der Einfluß von Serumfraktionen auf die Fettsäuresynthese}

\section{Mitteilung: Arbeitsveise für in-vitro-Untersucbungen mit dem löslichen Enzyntsystem der Hühnerleber ${ }^{1}$ )}

Von D. Eberhagen und W. Seitz

Technische Mitarbeit B. WrrmmanN

\author{
Aus der Medizinischen Poliklinik der Universität München (Direktor: Prof. Dr. W. Seitz)
}

(Eingegangen am 28. Februar 1966)

\begin{abstract}
Es wird eine vereinfachte Arbeitsweise zur Gewinnung des extramitochondrialen, fettsäuresynthetisierenden Hühnerleberenzymsystems als stabiles Trockenpräparat und zur Bestimmung seiner Aktivität beschrieben. Auf diese Weise wurden die mit Hilfe der präparativen trägerfreien Elektrophorese gewonnenen Fraktionen eines Normalserums und eines Diabetikerserums auf ihre Beeinflussung des Enzymsystems hin untersucht. Es fanden sich eine den ${ }^{14} \mathrm{C}$-Einbau aus Acetat in langkettige Fettsäuren fördernde und mehrere hemmende Fraktionen. Die Ergebnisse wurden mit Versuchen verglichen, die mit dem entsprechenden Enzymsystem der Taubenleber durchgeführt wirden.
\end{abstract}

A simplified method is reported for isolating and determining the activity of a stable dry preparation of the extramitochondrial, fatty acid-synthetising chicken liver enzyme system. Fractions obtained by preparative carrier-free electrophoresis from normal and diabetic serum were tested for their influence on the enzyme system. The incorporation of ${ }^{14} \mathrm{C}$-acetate into long chain fatty acids was stimulated by one fraction and inhibited by several others. The results are compared with those obtained with the corresponding enzyme system from pigeon liver.

In einer vorausgehenden Arbeit (1) war über die invitro-Beeinflussung des langkettige Fettsäuren aus Acetat de novo synthetisierenden extramitochondrialen Enzymsystems der Taubenleber durch verschiedene elektrophoretisch gewonnene Blutserumfraktionen berichtet worden. Bei diesen Untersuchungen hatte sich im Serum gesunder Personen eine nichtdialysierbare, im elektrischen Feld rasch anodenwärts wandernde Substanz

1) Die Untersuchungen wurden von der Deutschen Forschungsgemeinschaft unterstützt. gefunden, die eine ausgeprägte stimulierende Wirkung auf die Fettsäuresynthese besaß und wahrscheinlich phosphorhaltig war. Weiterhin ließen sich zwei Fraktionen mit einer Hemmwirkung auf den ${ }^{14} \mathrm{C}$-Einbau in die langkettigen Fettsäuren nachweisen. Ihre elektrophoretischen Wanderungsgeschwindigkeiten lagen zwischen denen der Präalbumine und der Albumine. In den Seren von Diabetikern wurde zusätzlich noch eine Fraktion ermittelt, die im elektrischen Feld noch stärker als die synthesefördernde Substanz abgelenkt wurde und 